\title{
Topical Applications of Antioxidants for Atopic Dermatitis
}

\author{
Rajesh R. ${ }^{1}$, Anbumalar ${ }^{2}$, Nithya P. ${ }^{3}$, Jaikumar S. ${ }^{4}$ \\ ${ }^{1}$ Associate Professor, Department of Dermatology, Meenakshi Medical College Hospital and Research Institute, \\ Enathur, Kanchipuram, Tamilnadu, India, ${ }^{2}$ Assistant Professor, Department of Dermatology, Meenakshi \\ Medical College Hospital and Research Institute, Enathur, Kanchipuram, Tamilnadu, India, ${ }^{3,4}$ Associate \\ Professor, Department of Pharmacology, Meenakshi Medical College Hospital and Research Institute, Enathur,
} Kanchipuram, Tamilnadu, India

\begin{abstract}
Atopic dermatitis is a skin disease which majorly affects the children. It can be caused by dust, mites, pets and food allergens. Atopic dermatitis is induced by the allergens and ends up in the elevated level of Immunoglobulin G. People from the developing countries such as New Zealand and U.S are more prone to atopic dermatitis skin disease because of the low level of melanin production in their skin. This can be reduced by the use of natural compounds such as glutathione derived from plants. In this article, the functioning and active molecules of glutathione for atopic dermatitis are discussed.
\end{abstract}

Keywords: Skin disease, Atopic dermatitis, glutathione, IgG, melanin.

\section{Introduction}

Microorganisms play an important role in curing the skin infections like eczema which are caused by the microbes like bacteria, fungus and viruses. Patient with atopic dermatitis is infected with the pathogen Staphylococcus aureus and these kinds of bacteria does not require antibiotic for the treatment. Antibiotics are used for the lesions and oozing leakages in the skin for the patients. Antimicrobial peptide production deficiency in the skin of atopic dermatitis can lead to have a greater risk of getting viral infections such as pox viruses caused by Molluscum contagiosum produced round shaped blisters, pimples on the skin. Typical super infection on the skin is caused by another important virus called herpes virus. Herpes simplex virus causes infection spreads all over the face and in the chest region leads to vesicular eruption. Eczemic conditions in patients are recommended for antiviral treatment ${ }^{[1]}$.

\section{Corresponding Author:}

\section{Rajesh R.}

Associate Professor, Department of Dermatology, Meenakshi Medical College Hospital and Research Institute, Enathur, Kanchipuram, Tamilnadu, India e-mail: rajesh@mmchri.ac.in
Glutathione: Glutathione consists of thiol, compound having a multifunctional role in plants. Function of glutathione is recognized earlier, is based on the thiol-disulphide interaction with other compounds. It works by how glutathione is reduction and oxidation manner to give a disulphide form that is then recovered by the NADPH-dependent glutathione reductase enzyme to form glutathione. Role of glutathione in animals is established as a defensive mechanism in animals that is because of the enzyme, selenium dependent glutathione peroxidase production which works as a central pillar of animal metabolism. Glutathione works as antioxidant in animals ${ }^{[2]}$.

The analysis of GSH(Glutathione) is gaining great interest in the field of science, many method have been proposed for the production of glutathione. The experiment on liquid chromatography and enzymatic determination are most processed experiments. Capillary electrophoresis is also one of the best method to obtain glutathione, which is simple, fast and reproducible method to obtain and analyze the compound with low cost effective procedure ${ }^{[3]}$.

Superoxide Dismutase: Atopic dermatitis are formed by the association of oxidative stress and others intruders in the development of skin peptides and proteins. This superoxide dismutase is worth in reduction 
of oxidative stress in the development of Atopic dermatitis in children. This is possible through the use of topical antioxidants, such as SOD. It is referred as the primary antioxidant and also as a ubiquitous enzyme, knows to be the defensive agent anti-oxidant ${ }^{[4]}$. This enzyme shows catalytic activity induces the reaction in a fast manner helps in renewing the process. Mechanism of action is not common with other active compounds such as vitamin E, glutathione, and carotenoids they are compounds which are believed to be easy exhaustible without the possible renew abilities. This enzyme is capable of converting an enzyme superoxide anion to hydrogen peroxide by distracting the reaction of $\mathrm{O}_{2}$. Hence the ROS production and free radicals are converted by this superoxide dismutase enzyme. A very harmful reactive nitrogen species are also protected by the enzyme superoxide dismutase ${ }^{[4]}$.

Vitamin C: It is considered as one of the water soluble in all the Vitamins. Lipophilic are antioxidant $\mathrm{A}$ and $\mathrm{E}$. Vitamin $\mathrm{E}$ is considered as the bond breaking antioxidants, which helps preventing the skin from oxidative effects and unsaturated lipids present on the membrane of the cell. For skin regeneration, Vitamin $\mathrm{C}$ is mostly preferred. It plays an important role in neutralizing free radicals by giving hydrogen ions to free radicals, then the vitamin $\mathrm{C}$ changes as ascorbate after the donation process. Vitamin $\mathrm{C}$ not only prevents the skin from free radicals induces oxidative stress it also works as Co-factor of glutathione and Vitamin $\mathrm{E}$ in the causative agents of stress in the production of proteins for skin protection. One of the most effective scavengers is beta-carotene works well against the alkoxyl and peroxide free radicals ${ }^{[4]}$.

Vitamin E: Atopic Dermatitis patients are treated with vitamin $\mathrm{E}$ orally and given as transdermal. The random clinical trial has suggested to use vitamin $\mathrm{E}$ for the topical application of this skin infection after observing the inflammatory responses. To incorporate such micronutrients nanotechnology approaches are made to make the compounds bioavailable water soluble vitamins. Nano formulations with vitamin $\mathrm{E}$ have been utilized for the efficient delivery of skin retention nanotechnological approaches towards this issue are more advantageous. ${ }^{[5]}$

Astaxanthin: These studies are conducted in mice for the antioxidant activity using Astaxanthin, 3,3'-dihydroxy- $\beta$-carotene-4,4'-Dione, carotenoid obtained from an algal compound without the Vitamin
A. Astaxanthin work efficiently than the $\beta$-carotene and $\alpha$-tocopherol. It has many therapeutic agents and activity such as anti-cancer, anti-tumor and antiviral activity. ROS activity is controlled by astaxanthin and oviduct epithelial cells in bovine also the n-oxidative stress. Additionally, Astaxanthin inhibits the inflammatory mediator production by controlling the signaling pathway of nuclear factor-kB. It prevents the skin from ultraviolet (UV) induced photokeratitis in the topical administration of mice by decreasing the oxidative stress in the eye cornea region ${ }^{[6]}$. An antioxidant, which inhibits the inflammatory response are Vitamin A, C and E. Antioxidants that are produced during the maternity period are intended to have protection against the child prone to atopic dermatitis leads to asthma ${ }^{[7]}$.

Melatonin: Melatonins are well known for immunostimulation in allergic reactions. It is also known for its antioxidant and cryoprotective effect in the cell affected by inflammatory actions. Inflammatory diseases lead to the decrease in melatonin secretion and free radical production that worsen and increases the skin infection. The release of Pro-inflammatory mediators and vaso active agents and infiltration of mast cells leads to the skin disorders such as atopic dermatitis. The immunoglobulin $\mathrm{E}$ and interleukin-4 in the serum are increased during the atopic condition when the melatonin production is inhibited in the skin. Allergens in the bronchial region indicate the presence of Immunoglobulin E leads to asthma by the inhalation of allergens. It regulates the immune response and shows the smoothness in muscle. This anti-oxidant however, increase the activity of pro-inflammation in asthma tends to bronchial inconvenience. It also works as a sleep inducing agent in asthma patients, but not recommended for intake in regular basis ${ }^{[8]}$

Solanum tuberosum L.: Solanum tuberosum extracts inhibit the lesion formation in atopic dermatitis patients from the study carried out in NC/NGA mice. Skin irritation, itching ear bulging and scratching habits was inhibited by the use of extracts from Solanum plant role is to inhibit the inflammatory responsive cells reaction. Solanum tuberosum treated mice showed reaction against the Ig E serum level ${ }^{[9]}$.

Mast cells and activation of helper cells are found in the patients with atopic dermatitis. It is found to be associated with an increase in Th2 cytokine serum, including interleukin-4, interleukin-5, interleukin-10, and interleukin-13 productions are noticed in patients 
with this skin infection. The expression level of interferons is also reported in patients with atopic dermatitis. Antioxidants play a major role in inhibiting the cells with a skin infection and protect the skin from UV-rays. $\beta$-hexosaminidase is also recommended in research incorporated to the mice to check the reaction against atopic dermatitis ${ }^{[10]}$.

Furfuryl Palmitite: It is an ester based compound obtained when palmitic acid reacts with furfuryl it has remarkable oxygen quenching property. This antioxidant is low reactive in nature, consisting of no ionic charge, which helps in spreading through the dermis layer beneath the skin and damages the cytoplasmic and nuclear composition. These are one of the primary reasons in skin aging and other dermatitis issues and it protects the skin in spreading infection ${ }^{[11]}$.

Natural Antioxidants: A Phenolic compound plays a major role in photoaging derived from natural compounds present in plants. It is identified in flavonoids. Flavones, isoflavones, cinnamon compounds these are derived from berries which are rich in antioxidants. These entire compounds play a major role in protecting the skin from oxidative stress, inflammatory response, UV-damage to the cells. All these compounds and skin care regimes play a defensive mechanism in protecting the skin from those damages ${ }^{[12]}$.

Ethanolic Extract of Juglans regia: Juglans regia plant showed very good scavenging activity and confirmed by the DDPH scavenging assay. These plants will protect the skin from damages ${ }^{[13]}$. The impact of ROS in biological systems is that the level of body fluids, DNA and lipids are measured by the biomarkers helps in the identification of diseases. DNA oxidative damage and repair are identified by the biomarker Urinary 8-hydroxydeoxyguanosine (8-OHdG). Some of the antioxidants are derived from protein with advanced antioxidant property and glycation products ${ }^{[14]}$

Ferulic acid: The major phenolic compound present in the plants works against the skin infection are ([E]-3-[4-hydroxy-3-methoxy-phenyl] prop-2enoic acid) in the plant tissues. Biological properties and phenolic compounds protect the skin from atopic dermatitis. Glycosides, tannins, flavones and tannins are present in plants which safe guards the health of human in many ways. Benzoic acid and cinnamic acids are the major phenolic compounds which are responsible for the reduction of free radicals and oxidative stress in the $\operatorname{skin}^{[15]}$.

\section{Conclusion}

Skin infections are higher in children due to many reasons such as close contact; unhygienic approaches such as hand wash and healthy food intake are the major problem in children. Atopic dermatitis is caused by the bacterium and develops as a major intolerable skin infection. This can be controlled by the antioxidants which will reduce the oxidative stress and UV-rays caused damages to the skin. Many antioxidants, phenolic compounds play a greater role in controlling the skin related disorders. The daily intake of these natural compounds and topical administration to the skin prevents from major skin diseases.

\section{Ethical Clearance: NA}

Source of Funding: Meenakshi Academy of Higher Education and Research, Chennai, India

\section{Conflict of Interest: NA}

\section{References}

1. Thomsen SF. Atopic dermatitis: natural history, diagnosis, and treatment. International Scholarly Research Notices. 2014;2014.

2. Noctor G, Mhamdi A, Chaouch S, Han YI, Neukermans J, Marquez-Garcia BE, Queval G, Foyer $\mathrm{CH}$. Glutathione in plants: an integrated overview. Plant, cell \& environment. 2012 Feb;35(2):454-84.

3. J. Chil. Chem. Soc. v.53 n.3 Concepción sep. 2008, Journal of the Chilean Chemical Society versión On-line ISSN 0717-9707

4. Noctor G, Mhamdi A, Chaouch S, Han YI, Neukermans J, Marquez-Garcia BE, Queval G, Foyer $\mathrm{CH}$. Glutathione in plants: an integrated overview. Plant, cell \& environment. 2012 Feb;35(2):454-84.

5. J. Chil. Chem. Soc. v.53 n.3 Concepción sep. 2008, Journal of the Chilean Chemical Society versión On-line ISSN 0717-9707

6. Noctor G, Mhamdi A, Chaouch S, Han YI, Neukermans J, Marquez-Garcia BE, Queval G, Foyer $\mathrm{CH}$. Glutathione in plants: an integrated overview. Plant, cell \& environment. 2012 Feb;35(2):454-84.

7. J. Chil. Chem. Soc. v.53 n.3 Concepción sep. 2008, Journal of the Chilean Chemical Society versión On-line ISSN 0717-9707 
8. Noctor G, Mhamdi A, Chaouch S, Han YI, Neukermans J, Marquez-Garcia BE, Queval G, Foyer CH. Glutathione in plants: an integrated overview. Plant, cell \& environment. 2012 Feb;35(2):454-84.

9. J. Chil. Chem. Soc. v.53 n.3 Concepción sep. 2008, Journal of the Chilean Chemical Society versión On-line ISSN 0717-9707

10. Noctor G, Mhamdi A, Chaouch S, Han YI, Neukermans J, Marquez-Garcia BE, Queval G, Foyer $\mathrm{CH}$. Glutathione in plants: an integrated overview. Plant, cell \& environment. 2012 Feb;35(2):454-84.

11. J. Chil. Chem. Soc. v.53 n.3 Concepción sep. 2008, Journal of the Chilean Chemical Society versión On-line ISSN 0717-9707

12. Diehl C. A novel efficient and safe treatment for atopic dermatitis: topical superoxide dismutase (SOD). Dermatol. Res. Skin Care. 2017;1:1-7.

13. Teo CW, Tay SH, Tey HL, Ung YW, Yap WN. Vitamin E in Atopic Dermatitis: From Preclinical to Clinical Studies. Dermatology. 2020 Oct 16:1-2.

14. Yoshihisa $Y$, Andoh $T$, Matsunaga K, Rehman MU, Maoka T, Shimizu T. Efficacy of astaxanthin for the treatment of atopic dermatitis in a murine model. PLoS One. 2016 Mar 29;11(3):e0152288.

15. Oh SY, Chung J, Kim MK, Kwon SO, Cho BH. Antioxidant nutrient intakes and corresponding biomarkers associated with the risk of atopic dermatitis in young children. European journal of clinical nutrition. 2010 Mar;64(3):245-52.

16. Marseglia L, D’Angelo G, Manti S, Salpietro C, Arrigo T, Barberi I, Reiter RJ, Gitto E. Melatonin and atopy: role in atopic dermatitis and asthma.
International journal of molecular sciences. 2014 Aug; 15(8):13482-93.

17. Shim EH, Choung SY. Inhibitory effects of S olanum tuberosum L. var. vitelotte extract on 2 , 4-dinitrochlorobenzene-induced atopic dermatitis in mice. Journal of Pharmacy and Pharmacology. 2014 Sep;66(9):1303-16.

18. Mechesso AF, Lee SJ, Park NH, Kim JY, Im ZE, Suh JW, Park SC. Preventive effects of a novel herbal mixture on atopic dermatitis-like skin lesions in $\mathrm{BALB} / \mathrm{C}$ mice. BMC complementary and alternative medicine. $2019 \operatorname{Dec}$ 1;19(1):25.

19. Pigatto PD, Diani M. Beneficial Effects of Antioxidant Furfuryl Palmitate in Nonpharmacologic Treatments (Prescription Emollient Devices, PEDs) for Atopic Dermatitis and Related Skin Disorders. Dermatology and therapy. 2018 Sep 1;8(3):339-47.

20. Monti DM, Rigano MM, Monti SM, Peixoto HS. Role of antioxidants in the protection from agingrelated diseases. Oxidative medicine and cellular longevity. 2019 Jan 1;2019.

21. Rahman TU, Khan T, Khattak KF, Ali A, Liaquat W, Zaib MA. Antioxidant Activity of Selected Medicinal Plants of Pakistan. Biochem. Physiol. 2016;5:1-5.

22. Bertino L, Guarneri F, Cannavò SP, Casciaro M, Pioggia G, Gangemi S. Oxidative Stress and Atopic Dermatitis. Antioxidants. 2020 Mar;9(3):196.

23. Zduńska K, Dana A, Kolodziejczak A, Rotsztejn H. Antioxidant properties of ferulic acid and its possible application. Skin pharmacology and physiology. 2018;31(6):332-6. 